\section{NOGALAMYCIN ANALOGS HAVING IMPROVED ANTITUMOR ACTIVITY}

Sir:

Nogalamycin $(\mathbf{1})^{1,2)}$ is an anthracycline antibiotic which has activity against Gram-positive microorganisms and is an antitumor agent. ${ }^{3)}$ However, because of its rather modest activity and undesirable side effects, it never became a clinically useful agent. It appeared likely that modification of $\mathbf{1}$ would give compounds superior in some respects to the parent compound since this has already been achieved by conversion of 1 to 7-O-methylepinogalarol $(2)^{2,3)}$. As a consequence, a program of modification was initiated which has led to agents which have been shown to be superior to $\mathbf{1}$ and at least the equal of adriamycin, the present standard in antitumor chemotherapy, in antitumor activity., ${ }^{4,5}$ The present communication describes the conversion of $\mathbf{1}$ to nogalamycinic acid (3), nogamycin (4), and 7-O-methylnogarol (5), and preliminary data on their biological activity.

Solution of 1 in $0.53 \mathrm{~N} \mathrm{KOH}$ solution for 16 hours followed by acidification $\left(\mathrm{H}_{2} \mathrm{SO}_{4}\right)$ gave the free acid (3). The crude product contained considerable inorganic material, but it was suitable for conversion to $\mathbf{4}$. Purification of $\mathbf{3}$ by column chromatography on silica gel using gradient elution with $\mathrm{CHCl}_{3}-\mathrm{CH}_{3} \mathrm{OH}$ gave a red solid, mp $219 \sim 229^{\circ} \mathrm{C}$; $\mathrm{Rf}\left(\mathrm{CHCl}_{3}-\mathrm{CH}_{3} \mathrm{OH}-\right.$ $\left.\mathrm{H}_{2} \mathrm{O} ; 78: 20: 2\right) 0.25 ;[\alpha]_{\mathrm{D}}+456^{\circ}(c) 0.37, \mathrm{CH}_{3-}$ $\mathrm{OH}) ; \mathrm{UV}(\mathrm{EtOH}) \lambda_{\max } 236 \mathrm{~nm}(\varepsilon 39,950), 269(\varepsilon$ 21,350), $291 \mathrm{sh}(\varepsilon 8,700), 482(13,550)$; IR (Nujol) $3450,1670,1630,1595,1580,1290,1230,1215$, $1135,1095,1060,1015,980,920,855,830,780$, 763 , and $725 \mathrm{~cm}^{-1}$; mass spectrum $\mathrm{m} / \mathrm{e} 729$ (M $\left.-\mathrm{CO}_{2}\right) ; \mathrm{H}^{1} \mathrm{NMR}\left(\mathrm{CDCl}_{3}-\mathrm{CD}_{3} \mathrm{OD}\right) \delta 1.38(\mathrm{~m}$, $\left.9 \mathrm{H}, 3 \mathrm{CH}_{3} \mathrm{C}\right), 1.80\left(\mathrm{~s}, 3 \mathrm{H}, \mathrm{CH}_{3} \mathrm{C}\right), 3.15[\mathrm{~s}, 6 \mathrm{H}$, $\left.\left(\mathrm{CH}_{3}\right)_{2} \mathrm{NH}^{+}\right], 3.38,3.40,3.68\left(3 \mathrm{~s}, 9 \mathrm{H}, 3 \mathrm{CH}_{3} \mathrm{O}\right)$, $3.2 \sim 4.0(\mathrm{~m}, \mathrm{CHO}$, and $\mathrm{CHN}), 5.24,5.88(2 \mathrm{~d}$, $2 \mathrm{H}$, anomeric), 6.92, 7.47 (2s, $2 \mathrm{H}$, aromatic); ${ }^{13} \mathrm{C}$ NMR $\left(\mathrm{CDCl}_{3}-\mathrm{CD}_{3} \mathrm{OD}\right) \delta 16.4,19.3,24.6$, $31.5\left(4 \mathrm{CH}_{3} \mathrm{C}\right), 42.5\left[\left(\mathrm{CH}_{3}\right)_{2} \mathrm{~N}\right], 49.9,58.2,60.3$ (3 $\left.\mathrm{CH}_{3} \mathrm{O}\right), 62.5 \sim 85.9(10 \mathrm{C}, \mathrm{CHO}, \mathrm{CHN}), 97.3$, 100.2 (anomeric), $113.1 \sim 161.4$, (12C, aromatic), 178.9, 181.6, 191.4 (carbonyl). The analysis and mass spectra of $\mathbf{3}$ did not establish its molecular formula, but conversion to $\mathbf{2}$ by treatment at room temperature with methanolic hydrogen chloride $(0.4 \mathrm{~N})$ and determination of the molecular formula of $\mathbf{4}$ combined with physical data defined the structure as $\mathbf{3}$ except that it exists as a zwitterion rather than as the free acid indicated.

When 3 was dissolved in DMF at room temperature for 16 hours, $\mathrm{CO}_{2}$ was given off (identified as $\mathrm{BaCO}_{3}$ ) and $\mathbf{4}$ was formed. Evaporation of the solvent gave a crude residue purified by chromatography on silica gel using gradient elution with $\mathrm{CHCl}_{3}-\mathrm{CH}_{3} \mathrm{OH}$ (yield of $\mathbf{1}$ to 3 about $25 \%$ ). Recrystallization from $\mathrm{CH}_{3}-$ $\mathrm{COCH}_{3}-\mathrm{CH}_{3} \mathrm{OH}$ (85: 15) gave a red solid, mp $210 \sim 215^{\circ} \mathrm{C}$; Rf (solvent as above) $0.50 ;[\alpha]_{\mathrm{D}}+$ $273^{\circ}$ (c $0.923, \mathrm{CHCl}_{3}$ ); UV (EtOH) $\lambda_{\max } \mathrm{nm} 236$ $(\varepsilon 51,700), 259(\varepsilon 25,850), 290(\varepsilon 10,050), 478$ (s 16,100); IR (Nujol) 3500, 1670, 1630, 1575, $1295,1230,1110,1055,1005,920,890,838,778$, 702 , and $724 \mathrm{~cm}^{-1}$; mass spectrum $m / e 729 ;{ }^{1} \mathrm{H}$ NMR $\left(\mathrm{d}_{\tau}\right.$-DMF) $\delta 1.14,1.23,1.37,1.69(12 \mathrm{H}$, $\left.4 \mathrm{CH}_{3} \mathrm{C}\right), 2.07 \sim 2.38,2.83 \sim 3.0\left(\mathrm{~m}, 4 \mathrm{H}, 2 \mathrm{CH}_{2}\right)$, $2.42\left[\mathrm{~s}, 6 \mathrm{H},\left(\mathrm{CH}_{3}\right)_{2} \mathrm{~N}\right], 3.13,3.42,3.52(3 \mathrm{~s}, 9 \mathrm{H}$, $\left.3 \mathrm{CH}_{3} \mathrm{O}\right), 3.3 \sim 4.2(\mathrm{~m}, \mathrm{CHO}, \mathrm{CHN}), 4.95(\mathrm{~m}, 1 \mathrm{H}$, benzylic $\mathrm{CHO}), 5.32,5.68(2 \mathrm{~d}, 2 \mathrm{H}$, anomeric), 7.16, 7.32 (2s, $2 \mathrm{H}$, aromatic); ${ }^{18} \mathrm{C}$ NMR $\left(\mathrm{CDCl}_{3}\right)$ d15.2, 18.3, 24.2, $30.4\left(4 \mathrm{CH}_{3} \mathrm{C}\right), 30.8,44.1$ $\left(2 \mathrm{CH}_{2}\right), 41.5\left[\left(\mathrm{CH}_{3}\right)_{2} \mathrm{~N}\right], 48.7,59.0,61.4\left(3 \mathrm{CH}_{3} \mathrm{O}\right)$, $66.4 \sim 88.6$ (10C, CHO, CHN), 96.9, 99.8 (anomeric), $113.1 \sim 161.4$ (12C, aromatic), 179.7, 190.8 (carbonyl).

Anal. Calcd. for $\mathrm{C}_{37} \mathrm{H}_{47} \mathrm{NO}_{14}$ : C, 60.96; H, 6.55; $\mathrm{N}, 1.92$. Found: C, 58.55; H, 6.42; N, 1.94 .

Methanolysis of $\mathbf{4}$ with boiling methanolic hydrogen chloride $(0.4 \mathrm{~N})$ for about 2 hours gave 5. Purification by chromatography on silica gel

Fig. 1
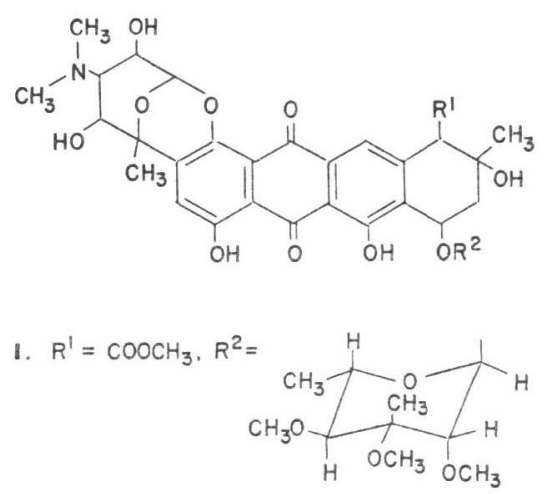

2. $\mathrm{R}^{\prime}=\mathrm{COOCH}_{3} \cdot \mathrm{R}^{2}=\mathrm{CH}_{3}$

3. $R^{1}=\mathrm{COOH}, \mathrm{R}^{2}=\mathrm{R}^{2}$ in I

4. $R^{1}=H, R^{2}=R^{2}$ in I

5. $R^{\prime}=H, R^{2}=\mathrm{CH}_{3}$ 
$\left(\mathrm{CHCl}_{3}-\mathrm{CH}_{3} \mathrm{OH} ; 95: 5\right)$ gave a $53 \%$ yield of a red solid; mp $248 \sim 253^{\circ} \mathrm{C}$; $\mathrm{Rf}$ (same solvent as above) $0.64 ;[\alpha]_{\mathrm{D}}+958^{\circ}\left(\mathrm{c} 0.163, \mathrm{CHCl}_{3}\right)$; UV (EtOH) $\lambda_{\max } \mathrm{nm} 235$ ( $\left.\varepsilon 41,200\right), 251(\varepsilon 25,500)$, 257 ( $\varepsilon 24,150), 290$ ( $\varepsilon 10,500), 479$ ( $\varepsilon 15,530)$; IR (Nujol) 3470, 1675, 1625, 1580, 1470, 1430, $1405,1385,1300,1230,1135,1115,1085,1065$, $1015,950,925,890,870,850$, and $790 \mathrm{~cm}^{-1}$; mass spectrum $m / e$ 541; ${ }^{1} \mathrm{H}$ NMR $\left(\mathrm{CDCl}_{8}\right.$ $\left.\mathrm{CD}_{3} \mathrm{OD}\right) \delta 1.45,1.73\left(2 \mathrm{~s}, 6 \mathrm{H}, \mathrm{CH}_{3} \mathrm{C}\right), 2.32 \sim$ $2.50,2.73 \sim 3.1\left(\mathrm{~m}, 4 \mathrm{H}, 2 \mathrm{CH}_{2}\right), 2.58[\mathrm{~s}, 6 \mathrm{H}$, $\left(\mathrm{CH}_{3}\right)_{2} \mathrm{~N}$ ], $3.60\left(\mathrm{~s}, 3 \mathrm{H}, \mathrm{CH}_{3} \mathrm{O}\right), 3.3 \sim 4.2(\mathrm{~m}, \mathrm{CHO}$, $\mathrm{CHN}), 4.83(\mathrm{~m}, 1 \mathrm{H}$, benzylic $\mathrm{H}), 5.82(\mathrm{~d}, 1 \mathrm{H}$, anomeric), 6.77, 7.25, (2s, $2 \mathrm{H}$, aromatic); ${ }^{13} \mathrm{C}$ NMR $\left(\mathrm{CDCl}_{3}-\mathrm{CD}_{3} \mathrm{OD}\right) \delta 23.9,30.0\left(2 \mathrm{CH}_{3} \mathrm{C}\right)$, $36.1,44.1\left(2 \mathrm{CH}_{2}\right), 41.6\left[\left(\mathrm{CH}_{3}\right)_{2} \mathrm{~N}\right], 57.9\left(\mathrm{CH}_{3} \mathrm{O}\right)$, $66.1 \sim 75.2$ (6C, $\mathrm{CHO}, \mathrm{CHN}), 97.6$ (anomeric), 112.6 161.1 (12C, aromatic), 179.7, 190.9 (carbonyl).

Anal. Calcd. for $\mathrm{C}_{28} \mathrm{H}_{31} \mathrm{NO}_{10}$ : C, 62.10; $\mathrm{H}, 5.78$; N, 2.59. Found: C, $62.21 ; \mathrm{H}, 5.94 ; \mathrm{N}, 2.66$.

Much more extensive biological data has been reported, ${ }^{4.5)}$ but as an indication of activity, Table 1 gives results obtained against P388 leukemia in mice.

\section{Acknowledgments}

The authors thank Dr. GARY L. NeIL and his collaborators for the biological data. This work was supported in part by Contract NO1-CM-43753 with the Division of Cancer Treatment, National Institutes of Health, Department of Health, Education and Welfare.

\section{P. F. WILEY \\ J. L. JOHNSON \\ D. J. HOUSER}

Research Laboratories

The Upjohn Company

Kalamazoo, Michigan 49001, U.S.A.

(Received February 15, 1977)
Table 1. Activity of nogalamycin analogs against mouse leukemia (P388)*

\begin{tabular}{c|c|c}
\hline Compound & $\begin{array}{c}\text { Dose } \\
(\mathrm{mg} / \mathrm{kg} / \text { day })\end{array}$ & $\%$ ILS \\
\hline $\mathbf{1}$ & 2.0 & 40 \\
$\mathbf{3}$ & 10 & 38 \\
$\mathbf{4}$ & 10 & 67 \\
$\mathbf{5}$ & 50 & 155 \\
Adriamycin & 1.0 & 57 \\
\hline
\end{tabular}

* All agents were administered ip days, 1, 5, and 9 after ip leukemia inoculation on day 0 except for adriamycin which was given on days $1 \sim 9$. Inoculum was $10^{6} \mathrm{P} 388$ cells/mouse given on day 0 . Median survivals were 10.2 10.7 days in the different experiments represented.

\section{References}

1) Bhuyan, B. K. \& A. Dietz: Fermentation, taxonomic, and biological studies of nogalamycin. Antimicr. Agents \& Chemoth. -1965: 836 844, 1966

2) Wiley, P. F.; R. B. Kelly, E. L. Caron, V. H. Wiley, J. H. Johnson, F. A. MacKellar \& S. A. Mizsak: Structure of nogalamycin. J. Amer. Chem. Soc. 99: 542 549, 1977

3) Bhuyan, B. K. \& F. Reusser: Comparative biological activity of nogalamycin and its analogs. Cancer Res. 30: 984 989, 1970

4) Neil, G. L.; C. L. Blowers, D. J. Houser, J. H. Johnson \& P. F. WILEY: Antitumor activity of some new analogs of nogalamycin. American Association for Cancer Research, Annual Meeting, Denver, Colorado, May 18 21, 1977

5) Li, L. H.; B. K. Bhuyan \& W. C. Krueger: Comparative biological and biochemical effects of nogalamycin and its analogs on L1210 mouse leukemia. American Association for Cancer Research, Annual Meeting, Denver, Colorado, May $18 \sim 21,1977$ 